\title{
Qual o índice de massa corporal de mulheres com disfunções dos músculos do assoalho pélvico que procuram tratamento fisioterapêutico?
}

\author{
What is the body mass index of women with pelvic floor muscles dysfunctions that seek \\ for physiotherapy treatment?
}

Fátima Faní Fitz', Thaís Fonseca Costa², Suellen Maurin Feitosa², Denise Rodrigues Yuaso ${ }^{3}$, Gabriel Andrade Alves $^{4}$, Marair Gracio Ferreira Sartori ${ }^{5}$, Manoel João Batista Castello Girão ${ }^{6}$, Rodrigo Aquino Castro ${ }^{7}$

\begin{abstract}
RESUMOISobrepeso e obesidade são relatados como importantes fatores de risco para desenvolvimento de disfunções do assoalho pélvico (AP) feminino. Assim, objetivou-se averiguar o índice de massa corporal (IMC) de mulheres com disfunções do AP que procuraram tratamento fisioterapêutico, e comparar com as estatísticas nacionais. Trata-se de um estudo observacional. Foram avaliados os prontuários de mulheres com disfunções do AP atendidas pelo Setor de Fisioterapia no período de 2004 à 2010, e incluídas todas as mulheres com a presença de algum sintoma de disfunção do AP. Calculou-se o IMC de 312 mulheres com disfunção do AP. A média de IMC foi de $28,1 \mathrm{~kg} / \mathrm{m}^{2}$. Dessas mulheres, cerca de $70 \%$ apresentavam sobrepeso ou obesidade, resultado maior do que o índice nacional de 59\%. A base fisiopatológica da relação entre obesidade e disfunções do AP está na correlação entre o IMC e a pressão intra-abdominal. A identificação do sobrepeso e da obesidade deve fazer parte dos programas de reabilitação do AP, uma vez que a redução do peso corporal pode contribuir para redução da severidade da disfunção. Com o presente estudo observa-se que as mulheres que procuram tratamento fisioterapêutico para disfunções do AP apresentam índice de obesidade maior do que a população feminina nacional.
\end{abstract}

Descritores I índice de massa corporal; obesidade; assoalho pélvico.

\begin{abstract}
I Overweight and obesity are reported as important risk factors for developing of female pelvic floor (PF) dysfunction. Thus, the objective was to verify the body mass index (BMI) of women with PF dysfunctions who sought physiotherapy treatment, and comparing it with national statistics. This is an observational study. There were evaluated the records of women with PF dysfunctions served by the Physiotherapy Service from 2004 to 2010, and included all women with the presence of any symptom of PF dysfunction. It was calculated the BMI of 312 women with PF dysfunction. The BMI mean was $28.1 \mathrm{~kg} / \mathrm{m}^{2}$. Approximately $70 \%$ of these women were overweight or obese, a value higher than the national rate of 59\%. The pathophysiological basis of the relationship between obesity and PF dysfunction is the correlation between BMI and intra-abdominal pressure. The identification of overweight and obesity should be part of the rehabilitation programs of the PF, since the reduction in body weight can contribute by reducing the severity of the disorder. With the present study it was observed that women seeking physiotherapy treatment for PF dysfunction have higher rates of obesity than the national female population.
\end{abstract}

Keywords I body mass index; obesity; pelvic floor.

\footnotetext{
Estudo desenvolvido no Ambulatório de Uroginecologia e Cirurgia Vaginal da Disciplina de Ginecologia Geral do Departamento de Ginecologia da Escola Paulista de Medicina da Universidade Federal de São Paulo (UNIFESP) - São Paulo (SP), Brasil.

'Fisioterapeuta, Especialista e Mestre em Ginecologia pelo Departamento de Ginecologia da UNIFESP - São Paulo (SP), Brasil. 2Fisioterapeuta, Especialista e Mestranda em Ginecologia pelo Departamento de Ginecologia da UNIFESP - São Paulo (SP), Brasil. ${ }^{3}$ Fisioterapeuta, Mestre e Doutoranda em Ginecologia pelo Departamento de Ginecologia da UNIFESP - São Paulo (SP), Brasil. ${ }^{4}$ Biomédico, Doutorando em Biologia estrutural e funcional pelo Departamento de Biofísica da UNIFESP - São Paulo (SP), Brasil. ${ }^{5}$ Médica, Doutora e Professora Associada, Livre-docente, Departamento de Ginecologia da UNIFESP - São Paulo (SP), Brasil. ${ }^{6}$ Médico, Doutor e Professor Titular do Departamento de Ginecologia da UNIFESP - São Paulo (SP), Brasil. ${ }^{7}$ Médico, Doutor e Professor Adjunto do Departamento de Ginecologia da UNIFESP - São Paulo (SP), Brasil.
} 


\section{INTRODUÇÃO}

O sobrepeso e a obesidade são relatados como importantes fatores de risco para o desenvolvimento de disfunções do assoalho pélvico (AP) feminino ${ }^{1-4}$.

A base fisiopatológica da relação entre obesidade e disfunções do AP é a correlação entre o índice de massa corporal (IMC) e a pressão intra-abdominal. Sugere-se que o sobrepeso e a obesidade podem estressar o AP pelo aumento de pressão intra-abdominal cronicamente ${ }^{1}$.

A incontinência urinária de esforço (IUE) estaria ligada ao aumento da pressão no AP, enquanto a hiperatividade da bexiga parece estar associada a respostas inflamatórias e químicas na bexiga urinária ${ }^{5}$. Além disso, obesidade vem sendo sugerida como fator de risco estabelecido para o prolapso de órgãos pélvicos ${ }^{6}$, bem como para sua progressão ${ }^{7}$.

Recentemente, a identificação do excesso de peso e da obesidade é sugerida como parte integrante dos programas de reabilitação do $\mathrm{AP}^{8}$. Assim, a redução de peso tem sido incluída no tratamento da incontinência urinária (IU) e do prolapso genital ${ }^{9,10}$, uma vez que a redução de 5\% do peso corporal contribui para redução da severidade da IU ${ }^{11}$.

Ambas as condições, obesidade e disfunções do AP, impactam negativamente na qualidade de vida das mulheres que as apresentam ${ }^{12}$. Com o aumento da prevalência da obesidade, verificou-se a necessidade de realizar um estudo da relação entre obesidade e IU para conhecer o perfil e melhorar a compreensão do papel desse fator de risco potencialmente modificável no desenvolvimento da IU nas pacientes brasileiras, em especial aquelas que procuram tratamento para as disfunções dos músculos do assoalho pélvico (MAP).

O objetivo do presente estudo foi averiguar o IMC de mulheres que procuraram tratamento fisioterapêutico para as disfunções do AP, bem como comparar com as estatísticas nacionais do IMC.

\section{METODOLOGIA}

Trata-se de um estudo observacional desenvolvido no Ambulatório de Uroginecologia e Cirurgia Vaginal da Universidade Federal de São Paulo (UNIFESP/EPM). Foram avaliados os prontuários de mulheres com disfunções do AP atendidas pelo Setor de Fisioterapia no período de 2004 a 2010. O estudo foi aprovado pelo Comitê de Ética em Pesquisa da UNIFESP - CEP $\mathrm{n}^{\circ}$ 60711. Incluíram-se mulheres com presença de disfunções do assoalho pélvico, diagnosticadas clinicamente pela equipe médica e que procuraram atendimento fisioterapêutico. Dentre os sintomas apresentados pelas mulheres, citam-se: incontinência urinária de esforço, incontinência urinária mista (IUM), urge-incontinência, bexiga hiperativa, enurese, cistite intersticial, prolapso genital, hipocontratilidade do detrusor e "sensação de vagina larga". As pacientes que necessitaram de exames complementares (com sintomas de incontinência urinária de esforço, incontinência urinária mista, urge-incontinência, bexiga hiperativa, cistite intersticial e hipocontratilidade do detrusor) passaram pelo estudo urodinâmico e por cistoscopia no caso de cistite intersticial. As pacientes com os demais sintomas (enurese, prolapso genital e sensação de vagina larga) foram diagnosticadas pela anamnese e exame físico.

Foi calculada a média de idade e do IMC, e a proporção das queixas apresentadas. De acordo com a World Health Organization (WHO), as mulheres foram assim classificadas: abaixo do peso $<18,5 \mathrm{~kg} / \mathrm{m}^{2}$; peso normal $18,5<24,99 \mathrm{~kg} / \mathrm{m}^{2}$; sobrepeso $\geq 25-29,99 \mathrm{~kg} / \mathrm{m}^{2}$; e obesidade $\geq 30 \mathrm{~kg} / \mathrm{m}^{2}$. Em seguida, foi realizada a comparação da classificação do IMC com os resultados recentes do IMC da população feminina brasileira segundo a $\mathrm{WHO}$ (disponível em: http://apps.who.int/bmi/index.jsp), com a utilização do teste $\chi^{2}$.

Por fim, comparamos as médias do IMC de pacientes com IUE e IUM com a utilização do teste $t$ de Student não pareado. Sempre foi adotado um nível de significância de 0,05 para o resultado ser considerado significante.

\section{RESULTADOS}

Um total de 345 prontuários foram analisados. Destes, incluíram-se 312. Foram excluídos 33 prontuários que não apresentavam os dados de altura e peso para cálculo do IMC. Para o cálculo da idade média foram considerados 309 prontuários que apresentavam esse dado. A idade média dessas mulheres foi de 53,6 $(12,8)$ anos. A Tabela 1 descreve a idade média das pacientes segundo a classificação do IMC da WHO. A Tabela 2 apresenta a proporção de mulheres segundo as queixas apresentadas. O IMC variou de 17,1 a $50,7 \mathrm{~kg} / \mathrm{m}^{2}$, com média de $28,1 \mathrm{~kg} / \mathrm{m}^{2}$. A Tabela 3 apresenta a proporção de mulheres atendidas segundo a classificação da WHO, bem como a comparação com os resultados do IMC nacional. 
Tabela 1. Idade média das pacientes segundo a classificação do índice de massa corporal da World Health Organization

$\begin{array}{lcc}\text { IMC }\left(\mathrm{kg} / \mathrm{m}^{2}\right) & \mathrm{n}=\mathbf{3 0 9} & \text { Média (DP) } \\ \text { Abaixo do peso/<18,5 } & 3 & 24,6(6,0) \\ \text { Peso normal/18,5<24,99 } & 90 & 53,3(13,9) \\ \text { Excesso de peso/ } \geq 25-29,99 & 107 & 55,4(12,9) \\ \text { Obesidade/ } \geq 30 & 109 & 52,8(10,7)\end{array}$

DP: desvio padrão

Tabela 2. Proporção de mulheres segundo as queixas apresentadas

$\begin{array}{lc}\text { Diagnóstico clínico } & \%(n=312) \\ \text { Bexiga hiperativa } & 2,5(n=8) \\ \text { Cistite intersticial } & 0,3(n=1) \\ \text { Enurese } & 0,3(n=1) \\ \text { Hipocontratilidade detrusor } & 0,3(n=1) \\ \text { IUE } & 48,7(n=152) \\ \text { IUM } & 41,3(n=129) \\ \text { Prolapso genital } & 0,6(n=2) \\ \text { Urge-incontinência } & 5,1(n=16) \\ \text { "Sensação de vagina larga" } & 0,6(n=2)\end{array}$

IUE: incontinência urinária de esforço; IUM: incontinência urinária mista

Tabela 3. Resultados do índice de massa corporal feminino nacional comparado com os resultados locais

$\begin{array}{lcr}\text { IMC }\left(\mathrm{kg} / \mathrm{m}^{2}\right) & { }^{*} \text { WHO } & \text { Resultados }(\mathrm{n}=312) \\ \text { Abaixo do peso/<18,5 } & 3,5 \% & 0,9 \%(\mathrm{n}=3) \\ \text { Peso normal/18,5<24,99 } & 54,8 \% & 28,8 \%(\mathrm{n}=90) \\ \text { Excesso de peso/ } \geq 25-29,99 & 43,0 \% & 35,2 \%(\mathrm{n}=110) \\ \text { Obesidade/ } \geq 30 & 16 \% & 34,9 \%(\mathrm{n}=109) \\ \text { **Excesso de peso/obesidade } & 59 \% & 70 \%(\mathrm{n}=219) \\ \text { Média de IMC } & - & 28,1 \mathrm{~kg} / \mathrm{m}^{2}(\mathrm{n}=312)\end{array}$

*Dados recentes referentes ao índice de massa corporal (IMC) da população feminina brasileira segundo World Health Organization (WHO) - Disponivel em: http:/lapps.who.int/bmi/index.jsp **Proporção de mulheres que se encontram acima do peso normal de acordo com a classificação do IMC

Além disso, ao fazermos o teste de ajuste das classes de IMC das pacientes desse estudo àquelas da população feminina brasileira, obtivemos uma diferença significante $\left(\mathrm{p}<0,001\right.$, teste $\left.\chi^{2}\right)$, mostrando, de fato, que as pacientes desse estudo têm, em média, maior IMC que a população feminina brasileira.

Na comparação entre as médias de IMC das pacientes com IUE e IUM, não foram obtidas diferenças significantes $(\mathrm{p}=0,263)$.

\section{DISCUSSÃO}

A média de idade das mulheres que procuraram atendimento fisioterapêutico para o tratamento de disfunções do AP, bem como as queixas principais estão de acordo com os tipos de queixas descritos em outros estudos ${ }^{13-15}$.

Atualmente descreve-se que mulheres com sobrepeso ou obesidade têm um risco maior de desenvolver $\mathrm{IU}^{4,14,16-18}$ e mulheres obesas apresentam medidas de IU mais severas do que mulheres incontinentes com peso normal ${ }^{19}$.

A obesidade é um problema que vem crescendo rapidamente, particularmente nos países desenvolvidos, onde sua prevalência nos últimos 30 anos tornou-se a principal epidemia de saúde pública ${ }^{20}$. A obesidade na idade adulta está associada a um aumento no risco de incapacidades ao longo da vida ${ }^{21}$, bem como tem sido associada com altas taxas de hipertensão, diabetes mellitus tipo II, hipercolesterolemia, aumento de risco para alguns tipos de câncer, aumento geral de morte prematura e outras condições crônicas ${ }^{20,21}$.

Em estudo realizado no Brasil nas regiões Nordeste e Sudeste, observou-se que mais da metade das mulheres dessas regiões com idade entre 40 e 79 anos têm sobrepeso $^{22}$. Em 2011, no Sul do Brasil, verificou-se que a prevalência de sobrepeso e obesidade $(64,3 \%)$ e de obesidade central $(44,1 \%)$ em usuárias de serviços do SUS é maior do que a encontrada na população geral e está associada a um aumento do número de agravos à saúde ${ }^{23}$.

Segundo os dados da WHO, 59\% das mulheres brasileiras encontram-se acima do peso normal (sobrepeso ou obesidade). Dentre as 312 mulheres atendidas em nosso serviço, os índices são maiores aos apresentados pela população feminina nacional.

Aston et al. encontraram resultados semelhantes em 161 mulheres com disfunções do assoalho pélvico atendidas por tratamento conservador. Eles notaram que a média do IMC foi $29,2 \mathrm{~kg} / \mathrm{m}^{2}$, maior do que a média nacional do reino Unido de $26,9 \mathrm{~kg} / \mathrm{m}^{2}$. Além disso, 75,3\% das participantes apresentavam sobrepeso ou obesidade em porcentagem maior do que a média nacional de 56,9\%, revelando que mulheres que procuram tratamento para as disfunções do assoalho pélvico possuem média maior de IMC do que a população feminina nacional ${ }^{8}$.

Em estudo semelhante, $74 \%$ das mulheres italianas com IU estavam com sobrepeso ou obesidade e $61 \%$ com disfunções sexuais, sendo que o IMC maior do que $30 \mathrm{~kg} / \mathrm{m}^{2}$ foi identificado como fator de risco para disfunção sexual feminina ${ }^{24}$.

Há evidências de que, além do IMC, a relação cintura-quadril e a obesidade abdominal podem ser fatores de risco independentes para a $\mathrm{IU}^{3}$. Em dois estudos brasileiros o IMC não foi associado à IU, porém, a probabilidade para IU aumentou com o aumento da circunferência abdominal. Em mulheres residentes no interior da região Sudeste, de 35 a 65 anos, a circunferência abdominal acima de $88 \mathrm{~cm}$ destacou-se como um dos fatores de risco para $\mathrm{IU}^{25}$. Em mulheres com mais de 
60 anos no Sul do Brasil, a probabilidade para IU foi de 1,98 para circunferência abdominal de $79-86 \mathrm{~cm}, 2,07$ para circunferência abdominal de $86-94 \mathrm{~cm}$ e 2,24 para circunferência abdominal maior que $94 \mathrm{~cm}(p=0,03)^{26}$. Em mulheres chinesas de 20 a 99 anos, circunferência abdominal $\geq 80 \mathrm{~cm}$ foi identificada como um dos fatores de risco para $\mathrm{IU}^{15}$.

Já em enfermeiras dos Estados Unidos, foi observada uma tendência significante de aumento do risco, severidade e frequência de IU com o aumento do IMC e circunferência da cintura. O IMC foi associado com urgência e IUM, mas não a IUE, e a circunferência da cintura, por sua vez, foi associada apenas com $\mathrm{IUE}^{27}$

É importante considerar que o IMC, apesar de ser uma variável conveniente com uma associação linear com o peso ${ }^{3}$, representa especificamente a gordura corporal total, enquanto a circunferência abdominal representa melhor a adiposidade central. A gordura corporal total e a adiposidade central refletem mecanismos diferentes que levam à IU, e investigações das relações independentes entre os vários tipos de IU podem fornecer percepções importantes a respeito dos seus papéis na etiologia da $\mathrm{IU}^{27,28}$.

Assim, é provável que o aumento do IMC exerça influência sobre a IU por meio de mecanismos sistêmicos, como por exemplo, pela ligação com o estresse oxidativo e resistência crônica a insulina ${ }^{29}$, fatores que podem causar danos vasculares ao assoalho pélvico e levar a disfunções do detrusor e músculos esfincterianos ${ }^{27}$. E o impacto da adiposidade abdominal na IU possa ser explicado através do impacto mecânico da adiposidade central na pressão intra-abdominal ${ }^{30,31}$, que passa a ser elevada cronicamente, resultando em pressão elevada sobre as estruturas de suporte da uretra e aumento no risco de desenvolvimento de $\mathrm{IUE}^{27}$. Podemos verificar que uma limitação do presente estudo foi a ausência da análise da medida da circunferência abdominal, que não é um procedimento de rotina em nosso serviço, limitando o conhecimento a respeito do seu papel na etiologia da IU na população estudada.

Outros autores verificaram o impacto do IMC e da circunferência abdominal sobre as pressões intra-abdominal e intravesical. Em mulheres com IU (86\% IUE), com sobrepeso ou obesas, a pressão intra-abdominal na capacidade cistométrica máxima aumentou $0,4 \mathrm{cmH}_{2} 0$ por unidade de $\mathrm{kg} / \mathrm{m}^{2}$ de IMC e $0,4 \mathrm{cmH}_{2} \mathrm{O}$ por aumento de $2 \mathrm{~cm}$ na circunferência abdominal. A pressão intravesical na capacidade cistométrica máxima aumentou $0,4 \mathrm{cmH}_{2} \mathrm{O}$ por $2 \mathrm{~cm}$ de aumento na circunferência abdominal, mas não foi associada ao $\mathrm{IMC}^{13}$.

A obesidade e o aumento do IMC estão relacionados à elevação do pico máximo de pressão intravesical gerado pela tosse (CIPP), e a redução do IMC está relacionada à menor $\mathrm{CIPP}$, o que pode sugerir que a perda de peso pode reduzir a incontinência pela modulação do CIPP ${ }^{32}$.

Estudos demonstram que a redução do peso leva a significante diminuição dos episódios de IU, da noctúria, melhora na qualidade de vida e decréscimo da pressão intravesical ${ }^{11,33}$.

Subak et al. revelaram que o grupo de mulheres que perderam em média $8 \%$ do peso inicial apresentou redução de no mínimo $70 \%$ na frequência de todos os episódios de $\mathrm{IU}^{33}$. De forma semelhante, Wing et al. afirmam que mulheres que perderam de 5 a $10 \%$ do seu peso corporal tinham 3,7 vezes mais chances de alcançar 70\% de redução dos episódios de IU total comparado com as mulheres que ganharam peso em 12 meses, e 2,4 vezes mais chances em 18 meses $^{34}$.

Os estudos citados ${ }^{11,33-35}$ suportam a perda de peso como opção de tratamento para mulheres com sobrepeso ou obesas e incontinentes, pois a redução dos episódios semanais de IU de 50 a $60 \%$ com a perda de peso é comparável com a melhora observada em estudos com terapias comportamentais e com terapias farmacológi$\operatorname{cas}^{35}$. Porém, Kudish et al. $^{7}$ observaram que a redução de peso não parece estar associada à regressão significativa do prolapso de órgãos pélvicos, sugerindo que o dano no assoalho pélvico, relacionado ao ganho de peso, pode demorar mais tempo para regredir ou ser irreversível.

De acordo com os resultados do presente estudo, verifica-se que mulheres com disfunções do assoalho pélvico apresentam índices de sobrepeso e obesidade maiores do que a média nacional. Assim, cabe aos profissionais de saúde identificá-las como grupo de alto risco para o desenvolvimento de disfunções do assoalho pélvico, e utilizar as vias para redução de peso como parte integrante na gestão dessas disfunções ${ }^{8}$.

\section{CONCLUSÃO}

No presente estudo observou-se que mais da metade das mulheres que procuram tratamento para disfunções do assoalho pélvico apresentam excesso de peso e obesidade com índices acima do observado na população feminina nacional. 


\section{REFERÊNCIAS}

1. Lawrence JM, Lukacz ES, Liu IL, Nager CW, Luber KM. Pelvic floor disorders, diabetes, and obesity in women: findings from the Kaiser Permanente Continence Associated Risk Epidemiology Study Diabetes Care. 2007;30(10):2536-41.

2. Erekson EA, Sung VW, Myers DL. Effect of body mass index on the risk of anal incontinence and defecatory dysfunction in women. Am J Obstet Gynecol. 2008:198(5):596.e1-e4.

3. Hunskaar S. A systematic review of overweight and obesity as risk factors and targets for clinical intervention for urinary incontinence in women. Neurourol Urodyn. 2008;27(8):749-57.

4. Subak LL, Richter HE, Hunskaar S. Obesity and urinary incontinence: epidemiology and clinical research update. J Urol. 2009;182(6 Suppl):S2-7.

5. Agur W, Rizk DE. Obesity and urinary incontinence in women: is the black box becoming grayer? Int Urogynecol J. 2011;22(3):257-8.

6. Jelovsek JE, Maher C, Barber MD. Pelvic organ prolapse. Lancet. 2007;369(9566):1027-38.

7. Kudish BI, Iglesia CB, Sokol RJ, Cochrane B, Richter HE, Larson J, et al. Effect of weight change on natural history of pelvic organ prolapse. Obstet Gynecol. 2009:113(1):81-8.

8. Aston BL, Sheehan L, Mawu G, Roberts C, Barnick C. Do women seeking treatment for pelvic floor dysfunction have higher than average BMl'S? In: Oral Presentations - 36 th Annual IUGA Meeting, Lisbon, Portugal, 28 June - 2 July 2011. Int Urogynecol J. 2011:22(Suppl 1):S1-195 p. 130.

9. Hagen S, Stark D, Glazener C, Sinclair L, Ramsay I. A randomized controlled trial of pelvic floor muscle training for stages I and II pelvic organ prolapse. Int Urogynecol J Pelvic Floor Dysfunct. 2009;20(1):45-51.

10. Resende APM, Stüpp L, Bernardes BT, Franco GR, Oliveira E, Girão MJBC et al. Prolapso genital e reabilitação do assoalho pélvico. Femina. 2010;38(2):101-04

11. Auwad W, Steggles P, Bombieri L, Waterfield M, Wilkin T, Freeman R. Moderate weight loss in obese women with urinary incontinence: a prospective Iongitudinal study. Int Urogynecol J Pelvic Disfunct. 2008;19(9):1251-9.

12. Sirls LT, Tennstedt S, Albo M, Chai T, Kenton K, Huang L, et al. Factors associated with quality of life in women undergoing surgery for stress urinary incontinence. J Urol. 2010;184(6):2411-5.

13. Richter HE, Creasman JM, Myers DL, Wheeler TL, Burgio KL, Subak LL, et al. Urodynamic characterization of obese women with urinary incontinence undergoing a weight loss program: the Program to Reduce Incontinence by Diet and Exercise (PRIDE) trial. Int Urogynecol J Pelvic Floor Dysfunct. 2008:19(12):1653-8.

14. López M, Ortiz AP, Vargas R. Prevalence of urinary incontinence and its association with body mass index among women in Puerto Rico. J Womens Health (Larchmt). 2009;18(10):1607-14.

15. Zhu L, Lang J, Liu C, Han S, Huang J, Li X. The epidemiological study of women with urinary incontinence and risk factors for stress urinary incontinence in China. Menopause. 2009;16(4):831-6.

16. Hannestad YS, Rortveit G, Daltveit AK, Hunskaar S. Are smoking and other lifestyle factors associated with female urinary incontinence? The Norwegian EPINCONT Study. BJOG. 2003:110(3):247-54.

17. Waetjen LE, Liao S, Johnson WO, Sampselle CM, Sternfield B, Harlow $\mathrm{SD}$, et al. Factors associated with prevalent and incident urinary incontinence in a cohort of midlife women: a longitudinal analysis of data: study of women's health across the nation. Am J Epidemiol 2007:165(3):309-18.
18. Ham E, Choi H, Seo JT, Kim HG, Palmer MH, Kim I. risk factors for female urinary incontinence among middle-aged Korean women. J Women's Health (Larchmt). 2009:18(11):1801-6.

19. Richter HE, Kenton K, Huang L, Nygaard I, Kraus S, Whitcomb E, et al. The impact of obesity on urinary incontinence symptoms, severity, urodynamic characteristics and quality of life. J Urol. 2010;183(2):622-8.

20. Poylin V, Serrot FJ, Madoff RD, Ikramuddin S, Mellgren A, Lowry AC et al. Obesity and bariatric surgery: a systematic review of associations with defecatory dysfunction. Colorectal Dis. 2011;13(6):e92-103.

21. Flegal KM, Graubard BI, Williamson DF, Gail MH. Cause-specific excess deaths associated with underweight, overweight, and obesity. JAMA. 2007:298(17):2028-37.

22. Abrantes MM, Lamounier JA, Colosimo EA. Prevalência de sobrepeso e obesidade nas regiões Nordeste e Sudeste do Brasil. Rev Assoc Med Bras. 2003;49(2):162-6

23. Rosa MI, Silva FML, Giroldi SB, Antunes GN, Wendland EM. Prevalência e fatores associados à obesidade em mulheres usuárias de serviços de pronto-atendimento do Sistema Único de Saúde no sul do Brasil. Ciênc. saúde coletiva. 2011:16(5):2559-66.

24. Pace G, Silvestri V, Gualá L, Vicentini C. Body mass index, urinary incontinence, and female sexual dysfunction: how they affect female postmenopausal health. Menopause. 2009;16(6):1188-92

25. Oliveira JM. Prevalência da incontinência urinária e sua associação com a obesidade em mulheres na transição menopausal e após menopausa [Internet]. São Paulo (SP): Faculdade de Saúde Pública/ USP; 2010 [cited 2011 Sep. 25]. Available from: http://www.teses.usp.br/ teses/disponiveis/6/6136/tde-13102.010.141622/en.php

26. Krause MP, Albert SM, Elsangedy HM, Krinski K, Goss FL, daSilva SG. Urinary incontinence and waist circumference in older women. Age Ageing. 2010;39(1):69-73.

27. Townsend MK, Curhan GC, Resnick NM, Grodstein F. BMI, Waist circumference, and incident urinary incontinence in older women. Obesity (Silver Spring). 2008;16(4):881-6.

28. Spiegelman D, Israel RG, Bouchard C, Willett WC. Absolute fat mass, percent body fat, and body-fat distribution: which is the real determinant of blood pressure and serum glucose? Am J Clin Nutr. 1992:55(6):1033-44.

29. Fenster CP, Weinsier RL, Darley-Usmar VM, Patel RP. Obesity, aerobic exercise, and vascular disease: the role of oxidant stress. Obes Res. 2002:10(9):964-8

30. Bump RC, Sugerman HJ, Fantl JA, McClish DK. Obesity and lower urinary tract function in women: effect of surgically induced weight loss. Am J Obstet Gynecol. 1992:167(2):392-7.

31. Noblett $\mathrm{KL}$, Jensen JK, Ostergard DR. The relationship of body mass index to intra-abdominal pressure as measured by multichannel cystometry. Int Urogynecol J Pelvic Floor Dysfunct. 1997;8(6):323-6.

32. Fuganti PE, Gowdy JM, Santiago NC. Obesity and smoking: Are they modulators of cough intravesical peak pressure in stress urinary incontinence? Int Braz J Urol. 2011:37(4):528-33.

33. Subak LL, Wing R, West DS, Franklin F, Vittinghoff E, Creasman JM, et al. Weight loss to treat urinary incontinence in overweight and obese women. N Engl J Med. 2009;360(5):481-90.

34. Wing RR, Creasman JM, West DS, Richter HE, Myers D, Burgio $\mathrm{KL}$, et al.. Improving Urinary Incontinence in Overweight and Obese Women Through Modest Weight Loss. Obstet Gynecol. 2010;116(2 Pt 1):284-92.

35. Subak LL, Whitcomb E, Shen H, Saxton J, Vittinghoff E, Brown JS. Weight loss: a novel and effective treatment for urinary incontinence. J Urol. 2005:174(1):190-5. 